\title{
La intercooperación: experiencia centroamericana
} (Intercooperation: the Central American experience)

\author{
Roxana Sánchez Boza ${ }^{1}$ \\ Universidad de Costa Rica
}

Reclbído: 01.07.2014

Aceptado: 01.09.2014

Sumario: I. Introducción. II. La integración cooperativa en Centroamérica: papel de entes cooperativos regionales. III. Casos sobresalientes en la región centroamericana: Costa Rica. 3.1. Un caso concreto del Siglo XXI de Integración cooperativa costarricense: Grupo empresarial cooperativo. IV. Iniciativas de reforma en aras de mayor integración por sectores: sector de ahorro y crédito y sector mujer en Costa Rica. 4.1. Sector mujer. V. Casos sobresalientes en la región centroamericana: Guatemala y Honduras; Panamá y Nicaragua: encadenamientos. 5.1. Guatemala y Honduras; 5.2. Panamá y Nicaragua: encadenamientos. VI. Conclusiones. VII. Bibliografía.

Resumen: La integración cooperativa se inicia considerando la importancia de un trabajo ordenado e integrado dentro de cada cooperativa. Con el desarrollo del Cooperativismo en su perfil empresarial surge cambios en su forma operativa, y se integran nuevas formas de realizar la labor productiva como son la integración económica por medio de tendencias conocidas como empredurismo, encandenamientos, redes financieras y empresariales. Nuevos grupos cooperativos son visibilizados para ofrecerles oportunidades de desarrollo socio-empresarial, estos son los y las jóvenes y las mujeres cooperativistas. Diversos programas son impulsados regionalmente en Centroamérica, ya sea por medio de la Confederación de cooperativas de Centroamérica, el Caribe y Suramérica, o bien por entes no cooperativos que pertenecen a la Economía Solidaria.

Palabras clave: integración cooperativa, empredurismo, encadenamientos, participación de sectores por género y juventud, redes y región centroamericana.

Abstract: Cooperative integration started by considering the importance that an orderly and integrated work scheme had within each cooperative. The development of a business profile for Cooperativism gives rise to changes in the way it is operated, and new ways of performing productive work are in-

1 Correo electrónico: metanoia500@yahoo.es 
tegrated such as economic integration using new trends known as entrepreneurship, linkages, financial and business networks. New cooperative groups are made visible to offer them opportunities for social and cooperative development, which are represented by young male and female cooperative members. A number of programmes have been regionally implemented in Central America, either through the Cooperative Confederation of Central America, the Caribbean and South America, or by non-cooperative entities within Social Economy.

Key words: cooperative integration, entrepreneurship, linkages, sector participation by gender and youth group, networks and central-american region. 


\section{Introducción}

La región centroamericana forma parte de un mismo origen geográfico por ser una de las porciones de América de más reciente formación geodésica. Pese a ese origen, el desarrollo cultural de los países del istmo centroamericano ha tenido influencias diversas desde la colonización entre los mismos y al interno se encuentran culturas indígenas, españolas y africanas que han determinado una multiculturalidad en los pequeños espacios de cada uno de esos países.

Sumado a lo mencionado, también se encuentra una diversidad de sistemas políticos que por mucho tiempo hacían que imperase el sistema dictatorial en la mayor parte de los países centroamericanos, donde Costa Rica siempre se ha caracterizado por tener menos períodos políticos teñidos de dictadura y por haber apuntado a un sistema democrático basado en la educación y la seguridad social universal. Casos extremos en cuanto a la alfabetización presentan Guatemala con un mínimo de preparación escolarizada sumada a un cuarenta por ciento de población indígena con pocas oportunidades de acceder a la educación frente a Costa Rica donde un alto porcentaje de la población, asiste a la escuela, un porcentaje menor a la educación secundaria y un grupo importante a la educación técnica y universitaria.

En Centroamérica nos encontramos básicamente seis países, si bien Belice está en el Istmo centroamericano, la conexión no es estrecha por razones de su desarrollo independiente, muy reciente, y el idioma.

El mundo cooperativo es cada vez más extenso y complejo, tiene relaciones al interno y al externo de cada ente cooperativo y de éste con el resto de sus homólogos por la unión que ofrecen los Valores y Principios cooperativos y por las relaciones que según la rama de la actividad elegida se establecen con el resto de organizaciones de la sociedad y del mundo.

Más de un siglo de existencia del Cooperativismo, pocos años del establecimiento de la Intercooperación como sexto principio cooperativista. Aunque no estaba expresamente declarado, siempre ha existido como base fundamental del desarrollo del Movimiento Cooperativo mundial. Su texto incorporado en 1995, en Manchester, Inglaterra, comunica la función de las cooperativas como entes al servicio de sus miembros, por medio de una labor eficaz y llena de fortaleza fundamentada en el trabajo en equipo, en todos los ámbitos en que se desarrollan las cooperativas, en un sociedad: su domicilio social establecido en los estatutos, su integración regional dentro 
del país de origen, nacional y hasta internacional. La letra de tal principio es la siguiente:

«Sexto Principio: Cooperación entre cooperativas: Las cooperativas sirven a sus miembros más eficazmente y fortalecen el movimiento cooperativo trabajando de manera conjunta por medio de estructuras locales, nacionales, regionales e internacionales».

La gama de posibilidades de integración de entes cooperativos inicia como apunta Martínez Charterina ${ }^{2}$ desde la misma familia y la cooperativa base extendiéndose a entes de segundo y ulterior grado. Mora $^{3}$ nos ofrece un excelente desarrollo del principio donde se toman en cuenta las relaciones de representación, defensa de algunos entes cooperativos así como los de tipo cultural o financiero.

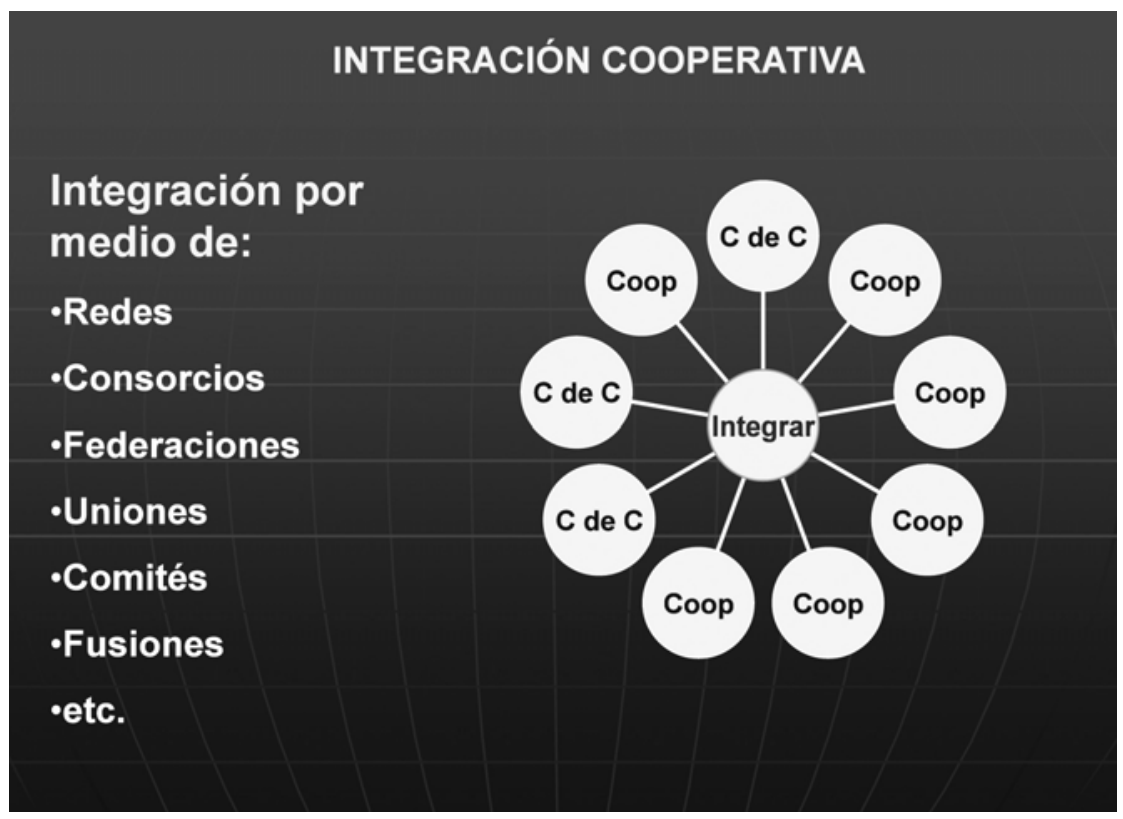

2 MARTíNEZ Ch, Alejandro en Sobre el principio de cooperación entre cooperativas en la actualidad N. ${ }^{\circ}$.46, 2012 (Ejemplar dedicado a: Las cooperativas construyen un mundo mejor), págs. 133-146.

3 MORA A. Dificultades y retos para competir eficientemente cuando no hay integración cooperativa, III Encuentro Educación Cooperativa, ACI-Américas, 2007.Lima, Perú, http://www.aciamericas.coop/Dificultades-y-Retos-para-competir. 
Mora 4 apunta algunas dificultades y retos de los entes de integración cooperativa y da algunas soluciones, indudablemente nos gustaría hacer un trabajo de campo que nos permitiera comprobar en cada país centroamericano la realidad de tales afirmaciones, o bien establecer las diferencias en su comportamiento real, sin embargo, consideramos que el análisis que plantea puede dar aportes significativos como punto de partida de establecer un estado de situación de tales entes en cada uno de países de la región, por ello transcribimos algunas de sus ideas y planteamientos.

El autor mencionado considera que existen dos aspectos fundamentales a discutir cuando se habla de organismos de integración, uno referido a los roles y funciones de dichos organismos, y sus miembros a todos los niveles, para evitar la duplicidad en el trabajo y evitar potenciales conflictos.

Y la dimensión, en términos de personal, infraestructura y costos operacionales, que el organismo de integración va a tener, siempre pensando que deben ser los miembros quienes tarde o temprano deben financiarla.

A partir de su visión de la integración cooperativa como forma de promover desarrollo empresarial, apunta Mora que falta definición de objetivos claros para visualizar los logros que se pretenden con la integración.

En algunos casos existe indefinición de roles y ámbitos de acción para los diferentes organismos de integración.

De tal modo que Mora propone fortalecer las organizaciones de base - pues los asociados y asociadas necesitan conocer sus derechos y tener acceso a ellos - y las organizaciones de integración de segundo y tercer grado. Promover la participación activa del cooperativismo en la economía nacional y regional (Incidencia). Incorporar los conceptos de eficacia, productividad y eficiencia, normas de calidad. El mejoramiento y modernización de la capacidad técnica, la capacidad gerencial y la capacidad dirigencial. Promoción de líderes jóvenes. La integración de la mujer en los procesos de decisión con los objetivos de elevar el ingreso económico de las mujeres, mejorar sus condiciones de vida y promover su participación más activa en las comunidades.

De la visión expuesta por Mora, compartida entre otros por Cordova, ambos ya citados y con conocimiento amplio del desarrollo del Cooperativismo Latinoamericano, el primero y centroamericano el segundo, nos complace encontrar múltiples ejemplos que desarrollan sus propuestas de mejora en la aplicación del principio de integración cooperativa.

4 ĺbid. 
Dejamos indicado, sin profundizar en este ensayo, que las formas tradicionales de integración cooperativa y que se encuentran en las leyes de cada país, se refieren a federaciones y uniones, en las cuales por haber sido el origen de las formas de integración dentro del Cooperativismo mundial, tienen múltiples objetivos, muy dispersos y que algunas ocasiones les ha impedido un desarrollo más adecuado a las necesidades de sus cooperativas representadas. Por otro lado, por la experiencia personal se puede apuntar que en muchas ocasiones esos entes llegan a tener tal autonomía en su funcionamiento, que los representantes pierden conexión con sus cooperativas de origen, al punto de no defender los intereses propios de la cooperativa que es el de los verdaderos dueños de la cooperativa: los socios.

En el caso de las federaciones y uniones de cooperativas se encuentran incorporadas a entes de representación y defensa del cooperativismo que se denominan confederación o consejos de cooperativas, los cuales encuentran reconocimiento de las leyes de cada país, y son los entes de decisión política, aquellos que generan política pública a favor de las cooperativas de cada país. ${ }^{5}$

Con la inserción cada vez más pronunciada del Cooperativismo en la Economía mundial, se dieron los consorcios cooperativos y ahora se habla de encadenamientos con base en el concepto de empredurismo. Existen muchas definiciones de encadenamiento, como es una figura de integración adoptada también en el cooperativismo aprovechamos la ofrecida por Pérez Sainz, con el objetivo de entender las propuestas que el Cooperativismo de algunos países de la región centroamericana ofrecen.

Pérez Sainz dice «Cuando tratamos de profundizar en el entendimiento del funcionamiento de la cadena, encontramos una mayor complejidad de las variables que actúan en el encadenamiento específico, con características específicas para productos o sectores de la industria, por ejemplo lenguajes, sistemas operativos, bases de datos, aplicaciones Internet, herramientas CASE, estándares, etc. Esto se vuelve más determinante cuando el objetivo es el Industrial Upgrading porque cabe preguntar mejoramiento de "qué» y «hacia dónde». Con respecto al «qué», existe una mayor claridad de limitaciones y necesidades de mejora del producto actual en función de requerimientos de reducción de costos vis a vis, mejoramiento de calidad del productoservicio, presentación, mercadeo, competencia relacionada con la inser-

5 Por ejemplo el artículo 136 de la Ley 4179 de Asociaciones Cooperativas (LAC) de Costa Rica crea el Consejo nacional de cooperativas como ente público no estatal y representante y defensor del cooperativismo costarricense. 
ción en cadenas globales. En cuanto al «hacia dónde», es precisamente donde el concepto de Industrial Upgrading demanda un esfuerzo de entendimiento de la Global Production Network (G P N ) 5 - Flagship / cadena global, de cómo las empresas locales se articulan con esta cadena global así como dentro de las locales, el papel, tipo de encadenamiento que las PyME logran o «tendrían» posibilidad de lograr» ${ }^{6}$.

En cuanto al empredurismo aprovechamos la entrevista al autor Kliksberg con motivo de la presentación de su último libro «Ética para empezar», porque reconoce que las cooperativas cumplen una importante función para disminuir desigualdades y la pobreza en el mundo, al respecto expresó?:

«Por esta razón, los procesos de intercooperación entre cooperativas de diferentes partes del planeta no son otra cosa que la promoción de procesos de desarrollo en aquellas zonas más desfavorecidas por las desiguales relaciones políticas y económicas entre el norte y el sur, agravadas en los últimos años por la globalización económica y las exigencias del Fondo Monetario Internacional y el Banco Mundial. La fórmula cooperativa tiene un elevado grado de implantación en algunas zonas del planeta, como por ejemplo Asia o Centroamérica. El apoyo a estas cooperativas no sólo fortalece al movimiento sino que, sobre todo, contribuye a generar alternativas al modelo económico imperante, generador de profundas desigualdades entre distintas regiones del mundo y al interior mismo de los países».

\section{La integración cooperativa en Centroamérica: papel de entes cooperativos regionales}

En la región centroamericana se da más trabajo conjunto por medio de las convocatorias en los ámbitos de capacitación, financiero y educativo en general. Esencialmente esas reuniones las propician dos entes como son la Confederación Latinoamericana de cooperativas de ahorro y crédito - COLAC - y la Confederación de cooperativas del Caribe, Centroamérica y Suramérica, una especializada en el tema financiero y

6 PÉREZ SÁINZ, JP. (EDITOR) et ut Encadenamientos globales y pequeña empresa en Centroamérica FLACSO (Sede Costa Rica); 1a. ed. San José, C.R. 2002. p 21.

7 SEMANARIO UNIVERSIDAD entrevista realizada por JAVIER CÓRDOBA MORALES a Kliksberg, B: «El neoliberalismo quiere expulsar la ética de la política y la economía», edición 12 de junio de 2013. 
la segunda con diversos temas que impulsa en los encuentros de cooperativistas esencialmente en la zona centroamericana y del Caribe.

COLAC en la próxima Convención financiera cooperativa latinoamericana, en el mes de setiembre 2014 contempla entre sus objetivos la integración como: un complemento de impecable presencia en los encuentros académicos. Demostrado está que el mundo político, social y económico están mirando los tamaños y las capacidades y la alta administración de las empresas; busca impactar al mercado mediante agrupamientos estratégicos, que no es otra cosa que unir recursos y tecnologías para fortalecer la competitividad.

Los dirigentes de COLAC consideran que las cooperativas no se pueden quedar atrás, y lo que ha sido tratado como un principio debe convertirse en una realidad, especialmente porque están dadas todas las condiciones para hacerlo sin temores y es algo que ya ha sido probado en los principales movimientos cooperativos del mundo.

Otro ente cooperativo de integración regional, con trabajo amplio en la región centroamericana y del Caribe, aunque también abarca la región de Sudamérica es la Confederación de Cooperativas del Caribe, Centro y Suramérica que cuenta con once programas regionales para ofrecer soluciones diferentes necesidades de las cooperativas del primer y ulterior grado.

1. Programa de Fortalecimiento y Desarrollo Institucional (PFDI) es para la Dirección general y administración de la CCC-CA, en aras de su consolidación organizativa y política integral. En cada país existe un Comité Nacional formado por las afiliadas locales que sirve de correa de transmisión y ejecutor de acciones nacionales.

2. Programa Regional de Capacitación Estratégica (PRCE) tiene el objetivo de contribuir al fortalecimiento doctrinario y al desarrollo de liderato cooperativista. En esa vía trabaja por elevar la capacidad de análisis y discusión de la dirigencia cooperativista a través de múltiples actividades, mecanismos e instrumentos. La capacitación se ofrece a distintos niveles: por temas, sectores, grupos dirigenciales o profesionales, por países y de apoyo a los proyectos.

3. Programa de Mejoramiento de la Calidad Cooperativa (PMCC) tiene como objetivo instalar capacidad y sistemas de gestión empresarial de calidad (SGC). El programa sensibiliza a organizaciones cooperativas sobre la necesidad de adoptar sistemas de gestión de calidad según la norma ISO 9001:2000, se acompaña con capacitación especializada para la adopción e implantación en su estructura de procesos ISO 9001:2000. Incluye a ejecuti- 
vos, dirigentes y personal en general, formando decenas de gestores y auditores internos de calidad que velan por el cumplimiento de los planes.

Luego ofrece asistencia técnica para implantar los procesos y hasta la auditoría externa de un ente internacional independiente que emite la certificación internacional de calidad. El $100 \%$ de las empresas apoyadas por CCC-CA en el PMCC han logrado su certificación, lo que es un récord reconocido.

4. Programa de Construcción de un Nuevo Modelo Organizacional Cooperativo (PCMO) tiene como objetivo contar con un grupo de cooperativas con estrategias y capacidad de gestión mejorada que sirvan de "vitrina» al cooperativismo. Se parte de la necesidad de revisión de su modelo, dictado por el contexto, para resolver distorsiones entre su razón de ser y lo que son. Se considera que el cooperativismo sigue vigente como instrumento de generación y distribución de riqueza y de gobernabilidad, cuando se pretende atribuir al mercado función de ordenador social.

De los sondeos realizados por la CCC-CA se ha encontrado que algunos sectores plantean como estrategia de mercadeo la «responsabilidad social» que la consideran parte del alma cooperativa así el éxito de muchas organizaciones cooperativas las obliga a revisar la relación entre su desarrollo como «asociación» y como «empresa».

El proyecto, con apoyo del SCC, sensibiliza al liderazgo sobre modelos de gestión, agregación de valor y estándares internacionales, aplica capacitación y asistencia técnica en las cooperativas del programa, y sistematizará la experiencia.

5. Programa de Mejoramiento de la Participación Cooperativa en el Desarrollo Rural (PMDR) busca crear capacidad instalada por organización y por país, generar bancos de información actualizados sobre el cooperativismo regional, y crear vínculos de cooperación, con el fin de fortalecer las organizaciones rurales cooperativas de Centroamérica y República Dominicana y así mejorar su contribución en el desarrollo de sus comunidades.

6. Programa Regional de Equidad Género y Generacional (PREG) El objetivo general es fomentar estrategias y acciones de participación igualitaria de género y generacional en el quehacer cooperativo. Para ello fortalece la presencia y acción de la dirigencia regional de las mujeres cooperativistas: Asegurando la capacidad estratégica y el análisis compartido del Comité regional de la mujer cooperativista (CRMC). Fomentando una participación visible de la dirigencia de las mujeres en los cónclaves coopera- 
tivistas de la región .Propiciando el cumplimiento de políticas de igualdad en las cooperativas y respaldando el desarrollo de una certificación de igualdad de género

Además propicia el desarrollo de los proyectos de mujeres cooperativistas y asegura la inclusión y cumplimiento de estrategias de género en los proyectos en ejecución.

7. Programa de Desarrollo Económico Local a través de Cooperativas (DELCOOP)

Con base en una alianza de CIF-OIT, CUDECOOP y la CCC-CA, funciona con el objetivo de desarrollar un programa de formación a distancia que prepare talentos capaces de generar procesos de desarrollo económico local. El programa es una plataforma de e-learning que utiliza modernas técnicas informáticas. El participante recibe un título de Gestor de desarrollo Económico Local, avalado por el sistema de las Naciones Unidas.

La formación de agentes multiplicadores de desarrollo económico local es responsabilidad de las cooperativas como cumplimiento del principio cooperativo de compromiso y responsabilidad hacia sus comunidades.

8. Programa de Desarrollo Gerencial Cooperativo (PDGC) tiene el objetivo de contribuir a incrementar la capacidad gerencial y empresarial en el movimiento cooperativo, por medio de módulos específicos: Planificación estratégica Mercadeo e inteligencia de negocios

9. Programa de Pasantías e Intercambio de Experiencias (PPIE)

Cuyo objetivo es compartir experiencias exitosas y conocer visiones modernizadoras así como referenciar criterios de calidad, desarrollo empresarial, fidelidad a los valores y principios cooperativos, sostenibilidad y responsabilidad con la comunidad.

10. Programa Regional de Información, Investigación y Comunicación (PR/C)

La CCC-CA dedica recursos a la investigación porque considera que es un instrumento esencial para que el cooperativismo cuente con información veraz y confiable para la correcta toma de decisiones, la búsqueda de un efectivo balance socio-empresarial y la definición de estrategias. En este programa se han realizado estudios dirigidos a: conocer la coyuntura del entorno, reconocer situación empresarial, mejorar la competitividad, estudiar productos o mercados, negociar compras conjuntas, conocer experiencias exitosas, definir estrategias y planes, posibilitar mayor valor agregado, construir modelos, ver opciones de comercialización, identificar cadenas de valor, estudiar viabilidades especiales, reducir costos. 


\section{Programa de Asistencia Técnica para el desarrollo (PATD).}

Cuenta con recursos humanos y un banco de consultores externos asociados con capacidad y sensibilidad cooperativa que permite brindar asistencia técnica para la gestión empresarial, disponible tanto a afiliadas como no afiliadas, con los siguientes servicios: Servicios corporativos ${ }^{8}$, capacitación $^{9}$, desarrollo organizacional ${ }^{10}$, mercadeo ${ }^{11}$.

Existen otras instancias que comprenden la región centroamericana, y entre los ejemplos del ámbito financiero se encuentran el Comité Regional de Cooperativas Financieras y Bancos Cooperativos de la Alianza Cooperativa Internacional en la Región de las Américas (COFIA) y la Plataforma Financiera para las Cooperativas de Ahorro y Crédito de la región Centroamericana, Panamá/México.

COFIA es un cuerpo especializado de la $\mathrm{ACl}$ Américas, formado como una organización sectorial y consultiva, no jurídico del Consejo de Administración Regional de ACl-Américas, constituido para elevar la competitividad empresarial de todos y cada uno de sus miembros.

Sus funciones son:

Ser un apoyo permanente al sector financiero cooperativo de la región.

Servir como instancia de consulta de los Órganos de ACl-Américas, en aquellos asuntos y problemas que atañen a las cooperativas financieras y bancos cooperativos de la región.

Ser un apoyo para el Consejo de Administración Regional de AClAméricas en la defensa y articulación con los entes internacionales emisores de normas de naturaleza contable, financiera, de supervisión y regulación que afectan a las cooperativas financieras y bancos cooperativos.

8 Entre los que se cuentan: Asesoría en Sistemas de Gestión de Calidad ISO 9001:2000. Asesoría en gestión de procesos y modelos de gestión. Formulación, evaluación, administración y monitoreo de proyectos. Procesos de modernización, reingeniería y reconversión. Organización de seminarios, conferencias, convenciones y talleres. Investigación y evaluaciones.

9 Se ofrecen cursos de capacitación especializada, en estándares internacionales; en estrategias de diferenciación.

10 Entre los contenidos están Procesos de definición y planificación estratégica, desarrollo de recursos humanos, incorporación de estrategias y políticas de género, asesoría personalizada en liderazgo y cualidades estratégicas, clima organizacional y cultura empresarial.

11 Propiciando estudios y definición de estrategias de mercado así como en capacitación en procesos de comercialización. 
Constituir el comité en un espacio de discusión, análisis e intercambio entre las cooperativas financieras y bancos cooperativos en las Américas, para elevar la competitividad de sus organizaciones.

Servir de enlace y facilitar la comunicación útil para todos sus miembros, permitiendo el flujo constante de información.

Promover el desarrollo empresarial y comercial de la actividad financiera cooperativa, en las Américas.

Apoyar el desarrollo de las iniciativas financieras cooperativas en los países de la región.

Facilitar un vínculo efectivo entre las cooperativas financieras y bancos cooperativos de las Américas.

Nutrir con su desarrollo las discusiones y debates del Comité Internacional de Bancos Cooperativos (ICBA) de la ACl.

Mantener una estrecha coordinación con el comité global correspondiente y con la Oficina Regional de la $\mathrm{ACl}$ en las Américas. ${ }^{12}$

Cordova ${ }^{13}$ informa en su análisis de la Integración Cooperativa sobre los objetivos de la RED FINANCIERA REGIONAL DE COOPERATIVAS DE AHORRO Y CRÉDITO DE CENTROAMÉRICA-CARIBE que busca el fortalecimiento de esa integración y con consciencia plena de los retos que el movimiento cooperativo tiene para posicionarse en el mercado regional, y asumen compromisos para resolverlos:

- proporcionando a EAES/CEPES-A, la información requerida para los proyectos regionales;

- asignando el personal técnico como referente para el apoyo a las consultorías según el área de especialización: remesas, tecnología, seguros, tarjetas, debito y crédito, capacitación, mercadeo, manuales administrativos, marco legal.

Así como asignar y registrar aportes de contrapartida para el funcionamiento del proyecto.

Objetivos que se traducen según Córdoba en resultados concretos, según los siguientes ejemplos de productos:

12 http://www.aciamericas.coop/Comite-Regional-de-Cooperativas-766.

13 Córdova, Héctor David, FEDECACES-El Salvador, Miembro Red Regional participación en Foro Diálogo: «La intercooperación como factor de integración empresarial, ante las exigencias de una economía abierta». "Caso: RED FINANCIERA REGIONAL DE COOPERATIVAS DE AHORRO Y CRÉDITO DE CENTROAMÉRICA-CARIBE» organizado por CEPES, Escuela de economía social de Andalucía España, en http://www.escueladeeconomiasocial.es/sites/default/files/Intercooperacion.RedFinanciera RegionaldeCooperativasdeAhorroyCreditodeCentroamericaydelCaribe.pdf. 


\section{TARJETA DÉBITO-CRÉDITO}

Las Federaciones conducen sus procesos de negociación con un mismo proveedor e intercambio tecnológico.

\section{REMESAS}

Las Federaciones acuerdan compartir su red de operadores internacionales, para extender el volumen de transferencias.

\section{SEGUROS}

Las Federaciones, a través de sus aseguradoras cooperativas, formularán oferta única para un tercer país, que no dispone de aseguradora cooperativa.

\section{MICROCRÉDITO}

En el nivel de la Red Regional, se acuerda definir su concepción del «microcrédito cooperativo» conectando con la economía social; distanciándose de mecanismos que ven a los pobres como negocio.

\section{TRANSVERSALES}

— Promoción de participación de la mujer en toma de decisiones.

- Desarrollo del sector de jóvenes cooperativistas.

- Participación del liderazgo dirigencial y técnico de la red en la Escuela de formación.

- Incidencia política, institucional y sectorial.

\section{Casos sobresalientes en la región centroamericana: Costa Rica}

En el año 2010 tuvo lugar el XII Congreso nacional cooperativo, el cual tiene como objetivo primordial establecer la política y los caminos a seguir por el cooperativismo costarricense. La metodología consistió en realizar foros según sectores económico, modelos de propiedad cooperativa: tradicional, autogestionaria o cogestionaria.

En cada foro se siguió un esquema por ejes y dentro de los mismos se revisaron formas de manifestación: liderazgo, innovación, gestión, competitividad y en el IV. EJE ESTRATEGICO DESARROLLO EMPRESARIAL se introdujo el abordaje del empredurismo. En cada foro el sector expuso sus problemas y sugirió soluciones para ser aprobadas en la 
asamblea de delegados de cooperativas que conforman el Consejo nacional de cooperativas. ${ }^{14}$

En relación con el tema EJE ESTRATEGICO DE RECTORIA POLITICA Y SOCIAL un vacio que surgió fue la indefinición del estilo de liderazgo que necesitan las Uniones y Federaciones. El cual fue parcialmente llenado cuando de innovación se trató porque se consideró de importancia la recomendación del organismo de integración correspondiente al proyecto propuesto para aprovechar una cartera especial si cumple con el requisito de novedad, de valor agregado y rentabilidad con responsabilidad ambiental.

En el tema de competitividad se solicitó la generación de encadenamientos cooperativos, basada en una política regional — dentro de Costa Rica - desarrollada por organismos cooperativos nacionales en coordinación con las Uniones y Federaciones respectivas.

En el III. Eje estratégico desarrollo económico local y territorial se establecieron tareas para fortalecer los organismos de integración cooperativa para que a su vez capaciten en forma permanente las cooperativas de base. Considerando que la investigación se desarrolle por medio de unidades de investigación para respaldo y apoyo a los emprendimientos cooperativos y establecer acciones para mejorar la gestión de las entidades de integración cooperativa, en los campos de innovación, gestión y competitividad cooperativa, donde el Instituto Nacional de Fomento Cooperativo juega un papel importante como asesor técnico $y$ ofreciendo el soporte financiero.

El IV. Eje estratégico desarrollo empresarial dio especial atención a los organismos de integración cooperativa, estableciéndoles tareas en coordinación con el INFOCOOP para impulsar estrategias de capacitación necesarias, en la renovación permanente, por medio de talleres, jornadas de capacitación, cursos, entre otros.

En cuanto a la gestión propusieron fomentar la creación de alianzas estratégicas entre cooperativas y organismos de integración cooperativa, lo cual no es novedoso pero si necesario profundizar en esas relaciones y ampliar posibilidades entre los actores cooperativos.

Finalmente el V. Eje estratégico articulación financiera consideró la necesidad de inyectar recursos a las uniones ya sea técnicos de capacitación, aporte financiero, para que estas puedan hacerle frente a las necesidades de sus asociadas en la generación de emprendimientos.

14 Es importante aclarar que no encontramos en la investigación realizada actividades similares en los otros países de la región centroamericana, aunque si hay abundancia de foros temáticos, como hemos visto con la cita de algunos en este ensayo. 
Lo anterior sin perder de vista la Identidad cooperativa cuando los organismos de integración desarrollen el modelo empresarial cooperativo.

Resalta en el aparte de innovación las propuestas para tener un conocimiento sólido de la situación del cooperativismo para impulsar proyectos, a saber.

1. Que los organismos de integración realicen un diagnóstico sobre el potencial y las capacidades que tienen sus afiliados y los territorios donde operan.

2. Que los organismos de integración realicen estudios que permitan identificar oportunidades de mercado con base a los potenciales de los afiliados y los territorios donde operan.

3. Diseñar un modelo alternativo de canalización de recursos de Banca de Desarrollo por medio de las Uniones y Federaciones, como organizaciones de segundo piso, en un modelo de crédito solidario dirigido a sus afiliados.

\subsection{Un caso concreto del siglo Xxı de integración cooperativa costarricense: grupo empresarial cooperativo}

En 2002 nace el Grupo Empresarial Cooperativo como una empresa $100 \%$ de capital costarricense. Conformado por la unión de diversas cooperativas, con el fin de apoyar su participación en el emprendimiento de actividades en diversos sectores de la economía nacional e internacional. Es una empresa exitosa de servicios múltiples, caracterizada por ser pionera en el modelo de emprendedurismo por medio del sector cooperativo, para el desarrollo de empresas exitosas.

Las cooperativas que participan son:

COOPENAE R.L. es una empresa modelo del sector cooperativo costarricense, reconocida por su impecable y prestigiosa trayectoria en el ámbito financiero. Destacada por brindar soluciones financieras y de mutualidad, rápidas y oportunas, para satisfacer las necesidades de sus asociados(as) y sus familias.

COOPEAGRI R.L. es una corporación cooperativa costarricense, catalogada como modelo en el ámbito nacional y latinoamericano, gracias a su diversificación que abarca áreas como: agrícola, industrial, comercial y financiera. Brinda soluciones a problemas de industrialización y comercialización del café de los pequeños y medianos caficultores de San Isidro de Pérez Zeledón.

CONAPROSAL R.L. es una empresa $100 \%$ costarricense, caracterizada por su compromiso social y empresarial, desarrollada en su diversificación y comercialización de productos como: ahorro y crédito a sus 
asociados, camarones, mariscos, sal, mangos, piña, papaya, pulpas y frutas.

También ha creado sus propias empresas: Grupo Tecnológico empresa orientada a la creación de soluciones tecnológicas. LKS Caribe empresa especializada en la implementación de Modelos de Gestión para apoyar el desarrollo del talento humano. Máxima empresa creadora de soluciones integrales en cuanto a servicios de seguridad, mantenimiento, mensajería y limpiezas para clientes corporativos y personales en temas de servicios generales. Asesorías y Cobros es una empresa cuya actividad principal es la recuperación de cartera desde hace ya más de una década. Su Objetivo Principal es recuperar su dinero. $L T I$ es una empresa Desarrolladora de sistemas de software especializado y creadora de soluciones en gestión empresarial.

\section{Iniciativas de reforma en aras de mayor integración por sectores: sector de ahorro y crédito y sector mujer en Costa Rica}

En el último Congreso cooperativo, el Foro preparatorio de cooperativas de ahorro y crédito presento un proyecto de reforma a la actual ley de cooperativas con el interés de integrar un cuarto sector a la asamblea de delegados del Consejo Nacional de cooperativas, actualmente integrado por grupos que no responden a la representación por volumen de negocios y número de socios, como es el caso del sector de intermediación financiera, iniciado y conocido en la ley a reformar como sector de cooperativas de ahorro y crédito .

Esos sectores son 1973 ley de creación del Consejo Nacional de Cooperativas (En el momento de nacer el CONACOOP y distribuir la representación del Plenario podemos deducir que la fuerza mayor estaba en las cooperativas agrícola-industriales, mientras que a las cooperativas de autogestión se buscó promoverlas y fortalecerlas con herramientas económicas o entregándoles bienes muebles e inmuebles para mejorar su patrimonio ${ }^{15}$.

15 La reforma a la Ley de Asociaciones cooperativas y creación del INSTITUTO NACIONAL DE FOMENTO COOPERATIVO, N. ${ }^{\circ} 4179$ y sus reformas, para el fortalecimiento del sector cooperativo de ahorro y crédito.

Artículo 1.- Reformase los siguientes artículos de la Ley de Asociaciones Cooperativas y Creación del Instituto Nacional de Fomento Cooperativo, N. ${ }^{\circ} 4179$, y sus reformas, para que en adelante se lean de la siguiente manera:

Artículo 138. - El Consejo Nacional de Cooperativas elegirá de su seno un presidente, un vicepresidente, un secretario y dos vocales, de tal suerte que representen los 
Los tres sectores contenidos en la Ley de asociaciones cooperativas son Agrícola industrial; Autogestión y Otras cooperativas que comprenden todas las cooperativas que no se encuentran en los otros dos sectores y se caracterizan por la multiplicidad de giros económicos.

intereses de los cuatro sectores. La asamblea se reunirá ordinariamente por lo menos cada tres meses y extraordinariamente cuando sea convocada por el presidente o por 10 delegados. El quórum lo formará la mitad más uno de los delegados.

Artículo 139.- El Consejo Nacional de Cooperativas será integrado mediante el siguiente procedimiento.

a) Se celebrarán cuatro asambleas separadamente: una de las cooperativas de autogestión, otra de las cooperativas de producción agrícola e industrial, una tercera de las cooperativas de ahorro y crédito y la cuarta de las demás cooperativas.

b) Cada cooperativa de primer grado, que se encuentre al día en el cumplimiento de sus obligaciones legales y que no se encuentre en causal o trámite de disolución, con el voto de los miembros de su consejo de administración y de los demás comités establecidos según sus estatutos, designará a un delegado, que deberá ser asociado, ante la asamblea que le corresponda. Las cooperativas participarán según la clasificación oficial que para ese efecto hará el Instituto Nacional de Fomento Cooperativo.

c) En las asambleas a que se refiere el inciso a) de este artículo, cada delegado tendrá derecho a un voto. No se admitirá voto por poder.

d) El quórum de estas asambleas será de la mitad más uno de los delegados presentes o de la totalidad Si una hora después de la fijada para la reunión no se hubiere completado ese número, se procederá válidamente a celebrar la asamblea con la asistencia de no menos del veinte por ciento $(20 \%)$ del total de delegados.

e) Cada una de las asambleas a que se refiere el inciso a) de este artículo, elegirá a diez representantes y tres suplentes. La cuarta asamblea, o sea la de las demás cooperativas, también elegirá a diez representantes y tres suplentes, pero ninguno de los sectores que la integren podrá elegir a más de tres representantes de federaciones o uniones nacionales. La elección en cada una de las asambleas según el inciso a), se hará por lista o papeletas, respetando la proporcionalidad de los votos obtenidos por cada papeleta en relación con el número de delegados por elegir. Las papeletas estarán constituidas por hombre y mujer en forma alternativa.

f) Las federaciones y uniones de cooperativas de ámbito nacional, debidamente inscritas y vigentes, tendrán derecho a designar un representante cada una ante el Consejo Nacional de Cooperativas.

g) Es deber del presidente del Consejo Nacional de Cooperativas convocar, con al menos treinta días de anticipación, a las cooperativas para las asambleas mencionadas en el inciso a), así como solicitar a las uniones y federaciones de cooperativas de ámbito nacional, la designación de sus representantes con al menos treinta días a la fecha de integración del plenario del CONACOOP.

Las asambleas de delegados de las cooperativas para elegir a los representantes que formarán el Consejo Nacional de Cooperativas y el nombramiento que hagan las federaciones y uniones, deberán realizarse cada cuatro años.

Artículo 141. - Para la elección de los cuatro representantes a la Junta Directiva del Instituto Nacional de Fomento Cooperativo, cada uno de los sectores representados en el Consejo Nacional de Cooperativas presentará una terna a conocimiento del plenario; de estas ternas se elegirá un representante de cada sector que será el miembro ante la junta Directiva del INFOCOOP. 
En 1987 se suple una laguna jurídica con la reforma del artículo 139 al introducir la participación de las uniones, federaciones y confederaciones a través del nombramiento no ya de delegados sino de representantes, con lo cual el universo de participación democrática del cooperativismo costarricense se ve enriquecido.

\section{IV.1. Sector mujer}

Dentro del enfoque del cooperativismo como actor político-social se propone con base en el eje de promoción: a) que en la conformación de los órganos de dirección se cumpla con la obligación legal de participación equitativa de la mujer, b) búsqueda de los mecanismos legales adecuados para que se cumpla la ley de paridad en los órganos de dirección.

\section{Casos sobresalientes en la región centroamericana: Guatemala y Honduras; Panamá y Nicaragua: encadenamientos}

\section{V.1. Guatemala y Honduras}

En Guatemala y Honduras una organización cooperativa andaluza denominada AMECOOP ha venido impulsando proyectos a favor de mujeres cooperativistas.

La Asociación de Mujeres Empresarias Cooperativistas de Andalucía, AMECOOP-Andalucía, es una entidad sin ánimo de lucro que existe desde el año2004 con la finalidad de crear un nuevo espacio para hablar de y con mujeres empresarias. Tiene como misión facilitar y crear espacios, procesos y herramientas a favor de sus socias, que les permita hacer aprendizajes colectivos e individuales, para conseguir protagonismo y presencia en el ámbito empresarial y público desde una conciencia de género grupal. El principal objetivo de Amecoop-Andalucía es la promoción y defensa de los valores humanos, sociales y profesionales de la mujer en su condición de empresaria

Acorde con este planteamiento de trabajo, AMECOOP está apoyando dos proyectos de desarrollo con cooperativas de mujeres en Centroamérica: uno en Guatemala, con la Federación de cooperativas artesanales, ARTEXCO, y otro en Honduras, con la Alianza de Mujeres de Cooperativas. ${ }^{16}$

16 MUJERES Y DESARROLLO: LA CONSTRUCCIÓN DE UNA RED. Por Ana Delso Atalaya. Coordinadora de AMECOO, integrante de la Comisión de Cooperación al Desarrollo de la Unión de Cooperativas Madrileñas de Trabajo Asociado (UCMTA). En Cua- 
Ambos proyectos basan en la capacitación la estrategia para mejorar la situación de las cooperativas y la situación de las mujeres dentro de las mismas. Las cooperativas integradas en ARTEXCO tienen entre sus asociados a más de un $50 \%$ de mujeres. Sin embargo, en los consejos rectores y órganos de representación las mujeres son minoría, así como en los puestos de gerencia de la cooperativa. La apuesta del proyecto es mejorar la capacidad gerencial de las mujeres y apoyar su participación en los órganos de toma de decisiones.

En un país como Guatemala, con un $60 \%$ de población indígena, la participación de las mujeres en las cooperativas está condicionada no sólo por las desigualdades de género, sino por las discriminaciones que sufre la población indígena. Muchas mujeres no hablan castellano, la educación de las niñas está siempre supeditada a todo lo demás, son las primeras que ayudan en la economía familiar, se privilegia la formación de los varones. La formación por tanto debe ser un eje fundamental para mejorar la presencia de las mujeres en todos los niveles de las cooperativas, lo que redundará sin duda en el beneficio de estas.

El proyecto sirve a su vez para fortalecer la presencia de una Unidad de Género dentro de la Federación de cooperativas, cuyas coordinadoras, dos mujeres de la etnia quiché y poptí, están realizando una labor inestimable para fortalecer la participación de las mujeres en las cooperativas y en las decisiones sobre hacia dónde tiene que ir el sector cooperativo artesanal en Guatemala, en concreto, y el movimiento cooperativo en general.

Por esta razón, los procesos de intercooperación entre cooperativas de diferentes partes del planeta no son otra cosa que la promoción de procesos de desarrollo en aquellas zonas más desfavorecidas por las desiguales relaciones políticas y económicas entre el norte y el sur, agravadas en los últimos años por la globalización económica y las exigencias del Fondo Monetario Internacional y el Banco Mundial. La fórmula cooperativa tiene un elevado grado de implantación en algunas zonas del planeta, como por ejemplo Asia o Centroamérica. El apoyo a estas cooperativas no sólo fortalece al movimiento sino que, sobre todo, contribuye a generar alternativas al modelo económico imperante, generador de profundas desigualdades entre distintas regiones del mundo y al interior mismo de los países.

Las subvenciones que con este objetivo están planteando las diferentes entidades públicas —estatales, regionales y locales - tienen 
como fin facilitar la creación de herramientas de cooperación al desarrollo desde distintos agentes sociales. El cooperativismo, como propuesta que busca el desarrollo y que contempla entre sus principios la intercooperación, no puede permanecer ajeno a este proceso y tiene que comprometerse, en la medida de sus posibilidades, con la mejora de las condiciones de vida en otras zonas y regiones, promoviendo la fórmula cooperativa como alternativa de desarrollo.

\section{V.2. Guatemala}

La Asociación Guatemalteca de Exportadores -AGEXPORT-, el Programa de las Naciones Unidas para el Desarrollo — PNUD_ y el Fondo Internacional del Desarrollo Agrícola — FIDA — han unido fuerzas para mejorar la capacidad de acceso a crédito, mercados, asistencia técnica especializada y al manejo empresarial sostenible para pequeños productores asociados. A pesar de la situación de crisis que atraviesa la economía mundial, existe una fuerte demanda por los productos de la región centroamericana que cumplan con los requisitos de calidad y denominación de origen por parte de los principales mercados mundiales-Europa, EEUU, Canadá, México y Asia-. Uno de los retos es entonces incorporar la producción de los pequeños productores para alcanzar una oferta productiva en volumen y calidad que cumpla con los estándares y las características de los mercados internacionales.

Uno de los logros de tales alianzas ha sido el reconocimiento a la Cooperativa de Mujeres 4 Pinos ubicada en San Juan Sacatepéquez, la cual con el apoyo del Programa de Encadenamientos Empresariales de AGEXPORT y la Embajada Real de Dinamarca a través de DANIDA, logró la certificación Global Gap, la cual las convierte en la primera cooperativa agrícola de mujeres certificada en el nivel centroamericano.

Además de ser la única cooperativa agrícola de mujeres, 4 Pinos, integrada por 340 socias, ahora se convirtió en la primera de la región, que logra certificar sus productos como los mejores del mundo. ${ }^{17}$

\section{V.3. Panamá y Nicaragua: encadenamientos}

Productores panameños de la región de Barú, en la provincia de Chiriquí, constituyeron una cooperativa buscando incrementar su com-

17 Publicado por Gladys NAZARENO en el Deguate.com, http://www.deguate.com/ artman/publish/gestion_articulos/primera-cooperativa-agricola-de-mujeres-a-nivel-centroamericano-recibe-certificacion-global-gap.shtml\#.U7NpA5pOVyQ 
petitividad. La cooperativa constituida por 80 productores panameños posee unas 800 hectáreas de las casi 6.200 sembradas con palma aceitera en la zona. Realizaron una inversión total de $\$ 7,8$ millones e inició operaciones con la planta extractora de aceite vegetal de palma africana, con una capacidad de procesamiento de 20 toneladas del fruto por hora. ${ }^{18}$

En el año 2010 se da un encadenamiento entre la actividad cooperativa productora de aceite crudo de palma y aceite seco de almendro producido por la Cooperativa de Productores de Palma de Chiriquí (Coopemapachi), de Panamá, la cual cerró una alianza con la nicaragüense Fraccionadora de Occidente, empresa del sector industrial.

Los cooperativistas vende materia prima a la Fraccionadora de Occidente y la empresa centroamericana le provee de productos para el envase de aceite en Panamá.

\section{Conclusiones}

Considerando la problemática del analfabetismo, gobiernos dictatoriales, guerras y grupos como los maras y dificultades económicas de los países centroamericanos, la Integración de las cooperativas es un medio que ofrece recursos y mejoramiento a muchos grupos de su población, gracias a la generación de empleo y la capacitación constante que desarrolla el cumplimiento del principio de Educación y formación cooperativa.

En general el desarrollo del Cooperativismo ha llevado a alianzas internas y externas dentro del mismo, plasmadas en el nacimiento de Federaciones, Uniones, Confederaciones, consorcios y otras figuras asociativas más o menos complejas.

Objetivos, visión y misión pueden tener confusión porque es tan amplia y extensa la tarea que deben cumplir los organismos cooperativos, que la tendencia inicial fue incorporar muchísimos y dísimiles objetivos a cumplir por el ente federativo de cooperativas.

El avance en la cultura económica y en la definición precisa de las necesidades en el campo político de representación y defensa así como empresarial de las cooperativas, les ha llevado a separar los momentos de ejercicio del poder político de aquellos momentos de toma de decisiones en relación con las alianzas, vinculaciones, encadenamientos con organizaciones de mucha experiencia empresarial.

18 Prensa.com,http://www.centralamericadata.com/es/article/home/Alianza_entre_ palmicultores_panamenos_e_industriales_nicaragenses, 25 de enero de 2010 . 
La decisión de la Alianza internacional cooperativa, en el año 1992 de establecer oficinas regionales en los diferentes continentes, ayudó a dar el empuje necesario para abrir caminos en las alianzas entre cooperativas de subregiones como la centroamericana y la sudamericana. Falta mucho camino por andar para lograr alianzas más abundantes de cooperativas de dos o más países. Sin embargo, en temas específicos existen redes, como la red financiera que hemos visto.

Existen entes internacionales, básicamente europeos como la Sociedad real de Noruega, Embajada Real de Dinamarca a través de DANIDA, entes de la ONU como el PNUD, cooperativas como AMECOOP en Andalucía y muchos otros entes dispuestos a entrar en alianzas con cooperativas para impulsar su desarrollo, de tal forma que la Integración de las cooperativas va más allá del Cooperativismo nacional o regional.

Costa Rica se propuso una interesante revisión del rol de las cooperativas y formuló solicitudes como respuesta a necesidades identificadas en el último Congreso nacional cooperativo.

Otros países de la región hacen los diagnósticos y las evaluaciones de sus necesidades y satisfacción por medio de encuentros de sectores cooperativos, eso sí indudablemente que el desarrollo de los programas de la Confederación de cooperativas de Centroamérica, el Caribe y Suramérica es de una riqueza invaluable, pues permite la integración al interno de cada país con los Comités Consultivos de las cooperativas miembros y en la región por medio de encuentros, investigaciones y acompañamiento en los proyectos de Identidad cooperativa, ayudando a marcar el norte ofrecido por los Valores y Principios cooperativos para que las cooperativas no se alejen de su debido cumplimiento. Aunado a lo anterior la CCC-CA colabora con la región buscando oportunidades de negocios, de capacitación y reúne experiencia para lograr una mejor evolución socio-económica del cooperativismo en las regiones americanas que atiende.

En resumen, estamos en proceso y progresamos en la integración cooperativa, existen organizaciones nacionales y regionales que ofrecen herramientas para lograrlo, en diferentes ámbitos, aunque el peso de muchas iniciativas del siglo pasado sigue guiando la actividad de muchos dirigentes y da la impresión de falta identificación de los objetivos reales, de pocos recursos humanos y financieros para lograrlo. La presencia del cooperativismo en cada país, obliga a los estados a disponer de fondos para su desarrollo; la solidaridad cooperativa como valor atrae cooperativas más avanzadas a tender la mano a las incipientes empresas cooperativas de otra región; hay abundancia de $\mathrm{OGN}$. $^{\circ} \mathrm{S}$ dispuesta a transferir su conocimiento de organización, encadenamientos y empredurismo para que otras cooperativas se beneficien. 


\section{Bibliografía}

ATALAYA, A. MUJERES Y DESARROLLO: LA CONSTRUCCIÓN DE UNA RED. Por Ana Delso Atalaya. Coordinadora de AMECOO, integrante de la Comisión de Cooperación al Desarrollo de la Unión de Cooperativas Madrileñas de Trabajo Asociado (UCMTA). En Cuadernos Mujer y Cooperativismo.N.22000,http://coceta.coop/cuadernos-mujer cooperativismo/Segundo/textos/ articulo16.htm.

CÓRDOVA, Héctor D, FEDECACES-El Salvador, Miembro Red Regional participación en Foro Diálogo: «La intercooperación como factor de integración empresarial, ante las exigencias de una economía abierta». "Caso: RED FINANCIERA REGIONAL DE COOPERATIVAS DE AHORRO Y CRÉDITO DE CENTROAMÉRICA-CARIBE» organizado por CEPES, Escuela de economía social de Andalucía España, en http://www.escueladeeconomiasocial.es/sites/default/files/Intercooperacion.RedFinancieraRegionalde CooperativasdeAhorroyCreditodeCentroamericaydelCaribe.pdf.

MARTÍNEZ Ch, Alejandro en Sobre el principio de cooperación entre cooperativas en la actualidad N. ${ }^{\circ}$. 46, 2012 (Ejemplar dedicado a: Las cooperativas construyen un mundo mejor), págs. 133-146.

MORA A. Dificultades y retos para competir eficientemente cuando no hay integración cooperativa, III Encuentro Educación Cooperativa, ACl-Américas, 2007.Lima, Perú, http://www.aciamericas.coop/Dificultades-y-Retos-paracompetir

NAZARENO, G artículo periodístico publicado en el De.Guate.com, http:// www.deguate.com/artman/publish/gestion_articulos/primera-cooperativaagricola-de-mujeres-a-nivel-centroamericano-recibe-certificacion-globalgap.shtml\#.U7NpA5pOVyQ

PÉREZ SÁINZ, JP. (EDITOR) et ut Encadenamientos globales y pequeña empresa en Centroamérica FLACSO (Sede Costa Rica); 1a. ed. San José, C.R. 2002. p 21.

Prensa.com,http://www.centralamericadata.com/es/article/home/Alianza_entre_palmicultores_panamenos_e_industriales_nicaragenses, 25 de enero de 2010.

SEMANARIO UNIVERSIDAD entrevista realizada por Javier Córdoba Morales a Kliksberg, B: «El neoliberalismo quiere expulsar la ética de la política y la economía» edición 12 de Junio de 2013. En http://www.aciamericas.coop/ Comite-Regional-de-Cooperativas-766. 